\title{
Jóvenes mexicanos y ciudadanía mundial para los objetivos del desarrollo sostenible
}

Mexican Youth and Citizenship for the Objectives of Sustainable Development

\author{
Alberto Farías Ochoa \\ Facultad de Letras \\ Universidad Michoacana de San Nicolás de Hidalgo \\ farias.ellh@gmail.com
}

Recibido: 5 de febrero de 2018 • Aprobado: 17 de marzo de 2018

Cómo citar este artículo: Alberto Farías Ochoa, "Jóvenes mexicanos y ciudadanía mundial para los objetivos del desarrollo sostenible", en Legajos. Boletín del Archivo General de la Nación, núm. 16 (mayo-agosto 2018), pp. 127-151.

\section{Resumen}

El presente artículo pone a disposición los resultados de los trabajos de contraste, y análisis, sobre la acepción del concepto de "ciudadanía mundial" por parte de jóvenes mexicanos matriculados en la universidad. Se muestra un recorrido que inicia con la revisión de las instituciones internacionales promotoras del concepto y su asimilación a cargo de los gobiernos en los sistemas educativos, continúa con el estudio de los documentos situados en las currícula escolares, para finalmente culminar en la evaluación por parte de los propios jóvenes, y sus referentes sobre el concepto.

Se trata de un diagnóstico tipo exploratorio, de enfoque cualitativo, que apunta a la construcción de un primer panorama para posteriores evaluaciones sobre jóvenes mexicanos y la ciudadanía mundial.

Palabras clave: ciudadanía mundial, jóvenes mexicanos, educación para la ciudadanía

\section{Abstract}

This paper presents the main results of the contrast and analysis work about the "Global Citizenship" concept in mexican university youth. The research departs from the review of those international institutions who promote the concept, and the governments who get it through the educational systems, the research continues with the analysis of the main documents contained in scholar curricula, and finally ends with the evaluation of the concept by the same mexican university students.

This is an exploratory diagnosis, with a qualitative view, which aims to the construction of a beginning panorama for further evaluations about mexican youth and global citizenship.

Keywords: global citizenship, mexican youth, education for citizenship 


\section{Introducción}

Las sociedades del nuevo milenio, principalmente las pertenecientes a los países en vías de desarrollo, han sido constantemente permeadas por el discurso urgente que apunta hacia los objetivos del desarrollo sostenible (ODs). Lo anterior, a partir de una perspectiva externa que pretende generar, desde las instituciones, líneas estratégicas a favor de los gobiernos y sus ciudadanos para afrontar, de manera coordinada, los retos más desafiantes del siglo xxi: los inherentes al calentamiento global, a la inequidad, al acceso a la educación y a las condiciones de violencia, entre otros.

En concordancia con los ops, durante la primera década del siglo xxi diferentes instituciones rectoras como la Organización de las Naciones Unidas para la Educación la Ciencia y la Cultura (unesco), El Banco Mundial y La Comisión Económica para América Latina y el Caribe (CEPAL), reiteraron la importancia sobre el concepto de "Ciudadanía Mundial", la cual se sugería fuera promovida por la estructura de la educación formal para su paulatina apropiación por parte de los nuevos ciudadanos de la región. En el año 2006 la UNESCO señalaba la necesidad de dar seguimiento y corroboración a la incorporación de la formación de ciudadanía mundial en los currículos de la educación formal; esto a través de las estructuras educativas de cada uno de los países, lo cual permitiría, a su vez, propiciar el contraste para la asimilación del concepto en los educandos. ${ }^{1}$

En la mayoría de los Estados de América Latina, en su condición de miembros de la Organización de las Naciones Unidas (ONU), se suscribieron acuerdos para incorporar, a través de las secretarías, ministerios y departamentos de educación de cada uno de los países, los contenidos para la formación de ciudadanía mundial dirigidos a los educandos y a los docentes. Al respecto, destaca el seguimiento generado en los casos de estudio de Colombia y Costa Rica, que ocupan

${ }^{1}$ Reimers, De Shano y Trevino, Where is the "Education", p. 42. 
las posiciones 95 y 65, respectivamente, en los índices de desarrollo humano, según el informe 2016 del Programa de las Naciones Unidas para el Desarrollo, ${ }^{2}$ el caso de estos dos países es relevante para el presente estudio debido a que en el año 2016 presentan condiciones similares a las de México en la apropiación del concepto de ciudadanía mundial en los currículos educativos; esto, después de una década transcurrida de compromisos entre gobiernos e instituciones internacionales rumbo a los oDs.

En el caso de Colombia y Costa Rica, la unesco enfatiza el buen trabajo que encuentra en el análisis de los currículos escolares reformados, principalmente destaca la incorporación para el conocimiento y discusión de conceptos clave como globalización, mundialización, internacionalización, derechos humanos y diversidad. No obstante lo anterior, sugiere también un rezago en comparación con los esfuerzos evidenciados en países asiáticos, considerados también en los mismos niveles de desarrollo, como el caso de Indonesia y Corea del Sur.

México, por su parte, ocupa el lugar número 77 en la tabla de índices de desarrollo humano, y ha incorporado en los planes de estudio para la educación básica varios elementos relacionados con el concepto de ciudadanía mundial, pero no el concepto como tal. De acuerdo con el documento oficial de la Secretaría de Educación Pública, ${ }^{3}$ los saberes vinculados con el concepto de ciudadanía mundial son atendidos de manera obligatoria en la tabla de contenidos de la asignatura de "Formación Cívica y Ética", dicha asignatura se desarrolla bajo la dirección del docente durante el segundo y tercer años de la llamada educación secundaria. En el entendido de que la educación secundaria es obligatoria en México, lo cual es avalado así en el Artículo 3o. de la Constitución Política de los Estados Unidos Mexicanos, se podría sugerir que todos los educandos deberán recibir dichos saberes en algún momento de su formación como ciudadanos adultos.

\footnotetext{
2 PNUD, Panorama general, p. 29.

${ }^{3}$ SEP, Plan de estudios 2011, 2011.
} 
En el contexto de los índices para el desarrollo destaca también, en el caso mexicano, la condición de continuidad de los jóvenes en el espacio escolarizado, y es que con base en los datos presentados por la Organización para la Cooperación y Desarrollo Económico (OCDE), solamente 38\% de los jóvenes mexicanos tienen acceso a la educación terciaria, ${ }^{4}$ lo que supone que el resto de los jóvenes no tendrán otra oportunidad más allá de la educación básica para la discusión de contenidos como ciudadanía mundial, interculturalidad, globalización, mundialización y otros relacionados. Así, se aprecia una brecha delicada entre los jóvenes del espacio escolarizado y aquellos que no pertenecen a éste, lo que circunstancialmente presenta una dificultad en los logros de la educación para la ciudadanía mundial, y por ende para el logro de los objetivos del desarrollo sostenible.

Con este panorama se gesta la inquietud que da pie al presente artículo, y es la de indagar las evidencias que refieren los jóvenes mexicanos favorecidos por el espacio escolarizado en asociación con el concepto ciudadanía mundial, esto, obviando la natural diferencia con aquellos jóvenes que ya no pertenecen al ámbito educativo. Aunado a lo anterior, interesa también conocer los métodos, escenarios y símbolos que dichos jóvenes emplean para la construcción de su noción de ciudadanía mundial; si los construyen a raíz de una currícula escolar o bien los desarrollan a través de otro tipo de prácticas en diferentes entornos; si sus referentes coinciden con las propuestas por parte de alguna organización rectora, o bien se reconocen capaces de formular sus propios conceptos. En concordancia con estos cuestionamientos, la pregunta central que orienta los trabajos de investigación para el producto del presente artículo se manifiesta de la siguiente manera:

¿Cuáles son las categorías asociadas al concepto de ciudadanía mundial que los jóvenes mexicanos matriculados en la universidad refieren, y en qué condiciones han apropiado los diferentes elementos relacionados con una acepción propia del concepto?

${ }^{4}$ OCDE, Panorama de la educación, p. 9. 
Dicho cuestionamiento central propicia, a manera de guía, un supuesto situacional sobre la condición de los jóvenes en relación con el concepto de ciudadanía mundial, y es: que los jóvenes universitarios sí tienen conciencia del concepto, al mismo tiempo que les interesan sus aristas relacionadas con la convivencia e inclusión de otros jóvenes; sin embargo, se pone en duda la eficiencia del espacio escolarizado como garantía para que se arraigue y reproduzca en todos los niveles de la escala social, lo que limita el privilegio de abonar a los objetivos del desarrollo sostenible como práctica solamente de algunos, aquellos que por su biografía de vida independiente de la escolarización han tenido la oportunidad de desarrollar la práctica entre pares.

\section{Comunicación, educación y práctica de la ciudadanía entre pares}

El concepto de "ciudadanía mundial" es, por sí mismo, complejo y difícil de acotar a ciertos derechos y obligaciones; en él las mismas organizaciones mundiales que fomentan el término reconocen un debate vigente. En relación con lo anterior, en este trabajo se concuerda con la postura de la propia unEsco cuando manifiesta que "El concepto de 'ciudadanía mundial' no puede expresarse en el plano jurídico, pero sí 'de otras maneras que pueden tener repercusiones importantes y profundas en el fomento de la participación cívica y las relaciones entre el ciudadano y el Estado". ${ }^{5}$ En este sentido, con la finalidad de propiciar una mirada flexible sobre el desarrollo del concepto, se contempla a los jóvenes en la práctica de la ciudadanía a través del acto comunicativo que propicia interacción, la que con la debida reproducción socializadora abona a un estatus dinámico de constante educación.

En dicho orden de ideas, se revisan en este apartado los conceptos de comunicación, educación y práctica, en el entendido de que es gracias a la conjugación de éstos que los jóvenes propician aprendizajes

5 Tawil, La educación para la "ciudadanía mundial", p. 2. 
relacionados con la ciudadanía, los que son significativos, que van más allá de la acumulación de información, y que generan diálogos problematizadores que derivan en nuevos significados, y que a su vez se someten a nuevos cuestionamientos de los practicantes. Así, a manera de ciclo, se genera una dinámica que, en el caso de algunos, permite la apropiación del concepto en complemento con la educación formal adquirida en las aulas.

\section{Comunicación}

Se considera la comunicación como elemento sustantivo de toda relación social, más allá de su connotación como un sistema de transmisión de mensajes o informaciones; se concibe como ese proceso básico para la construcción de la vida en sociedad. Es a través de las interacciones comunicativas desarrolladas entre las personas que se manifiestan los indicadores de la cultura como ejes organizativos de las experiencias humanas. "La comunicación consiste en un proceso simbólico que produce y reproduce patrones socioculturales compartidos". ${ }^{6}$ En este sentido se concuerda con tradiciones de estudio de la comunicación, como la perspectiva sociocultural, que genera interpretaciones sobre la manera en la que se elaboran los significados, normas, papeles y reglas a través del ejercicio de la comunicación.

Por su parte Marta Rizo puntualiza:

Es en la interacción comunicativa entre las personas donde, preferentemente, se manifiesta la cultura como principio organizador de la experiencia humana. La comprensión de la comunicación como telón de fondo de toda actividad humana se fundamenta en una perspectiva de corte sistémico, que implica considerar a la comunicación como un conjunto de elementos en interacción

\footnotetext{
${ }^{6}$ Fernández y Galguera, Teorías de la comunicación, p. 13.
} 
donde toda modificación de uno de ellos altera o afecta las relaciones entre otros elementos. ${ }^{7}$

En este tenor, podría comprenderse que todo lo que es comunicación es sociedad, y la comunicación se ejerce como un sistema regulador del proceso de civilización mediante el cual los sujetos paulatinamente se hacen dependientes de estos procesos, así pueden elegir sus relaciones con otros similares y dada la multiplicación de relaciones se constituye lo que se reconoce como sociedad.

Otro concepto vinculado al acto comunicativo es la interacción entendida como la acción recíproca entre dos o más agentes, en la que, independientemente de quién inicie esta acción, el resultado siempre será la modificación de los estados de los participantes, es decir la realidad para ambos, en pequeña o gran medida, habrá sido transformada; es el caso, por ejemplo, de la práctica de la ciudadanía.

\begin{abstract}
La interacción además de estar estrechamente relacionada con la comunicación en entornos educativos, se vincula también a los procesos de socialización en general, así como la manera como los actores de la comunicación construyen su diálogo y autoerigen en miembros de la comunidad. La socialización supone la capacidad de relacionarse con los demás, de incorporar las reglas al entorno, negociarlas y ajustarlas a sus necesidades. En términos comunicativos, la socialización supone que el sujeto cuenta con los mecanismos necesarios para enviar y recibir información, para interpretarla y significarla. Todo ello, porque el ser humano no se realiza en solitario sino en medio de otros individuos con quienes interactúa. ${ }^{8}$
\end{abstract}

\footnotetext{
${ }^{7}$ Rizo, "Interacción y comunicación", p. 3.

${ }^{8}$ Winking, en Rizo, "Interacción y comunicación”, p. 4.
} 


\title{
Interacción y proceso educativo
}

La interacción, cuando se da con sentido formativo, busca dejar huella en el actor que aprende, e influye en él en forma de conocimientos, o bien en forma de habilidades. Se entiende, entonces, que el proceso de enseñanza y aprendizaje genera cambios en los individuos participantes, quienes adoptan las transformaciones en una dinámica que favorece a sus intereses. En esta lógica, el aprendizaje es también el producto de un proceso activo de construcción, de una interacción social donde el sujeto aprende de otros y con los otros, desarrollando, a través de esa interacción, su inteligencia, construyendo e interiorizando nuevos conocimientos a lo largo de su vida. Concibiendo el aprendizaje como producto y resultado de la educación, se le da la importancia a esta última como un proceso continuado a través de las interacciones sociales en el desarrollo de las capacidades del sujeto.

\begin{abstract}
Como resultado de la interacción, el proceso de enseñanza-aprendizaje puede ser considerado como un proceso de comunicación, porque se desarrolla en el marco de relaciones de interacción. Las transferencias de habilidades, conocimientos y actitudes tienen lugar mediante el diálogo o interacción entre facilitadores y estudiantes, y el conocimiento de los estudiantes es juzgado por los docentes a través de juicios que desarrollan también un proceso comunicacional. ${ }^{9}$
\end{abstract}

La educación es "un proceso que dura toda la vida y en el que las personas adquieren y acumulan conocimientos, habilidades, actitudes y modos de discernimiento mediante las experiencias diarias y en relación con su medio ambiente". ${ }^{10}$ En un sentido más preciso, relacionado

${ }^{9}$ Rizo, "Interacción y comunicación", p. 7.

${ }^{10}$ Coombs y Ahmed, en Sarramona, Vázquez y Colom, Educación no formal, p. 12. 
con el análisis de las condiciones de aprendizaje del sujeto para la ciudadanía mundial, se coincide con la postura de Sirvent, ${ }^{11}$ quien sustenta que la educación ciudadana permanente se asienta sobre los siguientes principios teóricos y metodológicos:

a) La concepción de la educación como una necesidad permanente y como un derecho para todos los individuos y grupos sociales.

b) El reconocimiento de la capacidad de individuos y grupos para el aprendizaje y la transformación a lo largo de toda su existencia.

c) La consideración de la experiencia vital como punto de partida para procesos de aprendizaje continuo.

d) El reconocimiento de la existencia de múltiples formas y recursos educativos emergentes de una sociedad, que operan en la escuela y más allá de la escuela.

e) El supuesto de la potenciación de los recursos educativos a través de la construcción de una red o trama que los articule.

f) El reconocimiento de la importancia y la necesidad de la participación social en las cuestiones referidas a la educación y la democratización del conocimiento.

En continuidad con la perspectiva de Sirvent, y en relación con la concepción de la educación de jóvenes y adultos, ésta es concebida como aquel ámbito de conjunto de actividades relacionadas con las necesidades educativas correspondientes a las distintas áreas de la vida cotidiana del joven y del adulto. Se contemplan las actividades complementarias, pero determinantes para el ejercicio de la ciudadanía, posteriores a la educación inicial, así como las orientadas al trabajo, a la vida familiar, la vivienda, la salud, la recreación, la participación social y política, todas como posibilidad de desarrollo y optimización a lo largo de toda la vida. Incluye experiencias muy diversas que pueden

${ }^{11}$ Sirvent, "La educación de jóvenes y adultos", p. 40. 
ir desde la capacitación laboral hasta la formación de ciudadanía para la participación activa en la comunidad. ${ }^{12}$

Aunado a la perspectiva de Sirvent, se retoma de forma muy particular la manera en la que Freire destaca la importancia del diálogo como elemento vital de la actividad educadora, y es que el diálogo propicia dos dimensiones que son componentes indispensables de la palabra empleada por los participantes: la acción y la reflexión. $^{13}$ Señala que mediante el uso de la palabra se emplea ya una reflexión, y entonces se convierte la palabra en activismo, esto a diferencia de la llamada palabra inauténtica la que es privada de su dimensión activa y por tanto incapaz de transformar la realidad.

Interesa destacar al diálogo desde la concepción de Freire como elemento fundamental del acto educativo para la ciudadanía, como algo que va más allá que un simple medio de intercambio, como un fenómeno humano en el que se incorporan, o no, compromisos para la participación activa. Mediante el diálogo, la existencia humana no puede permanecer silenciada ante su realidad. Dicha realidad debe de ser pronunciada con aquellas palabras que, al incorporar acción y reflexión en sus intercambios, generen un proceso de transformación. "Existir, humanamente, es 'pronunciar' el mundo, es transformarlo. El mundo pronunciado, a su vez retorna problematizado a los sujetos pronunciantes, exigiendo de ellos un nuevo pronunciamiento". ${ }^{14}$

\section{La práctica como acto social}

Guando se parte del concepto de práctica como la acción que va más allá de hacer algo en sí mismo, es el hacer algo dentro de un contexto histórico y social otorgándole estructura y significado a eso que se hace. Lo que de acuerdo con Wenger es siempre una práctica social. Como

\footnotetext{
12 Sirvent, "La educación de jóvenes y adultos", p. 41.

${ }^{13}$ Freire, Pedagogía del oprimido, p. 105.

${ }^{14}$ Freire, Pedagogía del oprimido, p. 106.
} 
el caso de los esfuerzos que apuntan hacia una ciudadanía mundial acorde con los objetivos del desarrollo sostenible.

Este concepto de práctica incluye tanto los aspectos explícitos como los implícitos. Incluye lo que se dice y lo que se calla, lo que se presenta y lo que se da por supuesto. Incluye el lenguaje, los instrumentos, los documentos, las imágenes, los símbolos, los roles definidos los criterios especificados, los procedimientos codificados, las regulaciones y los contratos que las diversas prácticas determinan para una variedad de propósitos. ${ }^{15}$

En este sentido el proceso de participación en la práctica siempre implica el todo de la persona, actuando y conociendo al mismo tiempo, $\mathrm{y}$ en el que acción y conocimiento forman parte del mismo objetivo y no son determinados como elementos aislados en el aprendizaje de la ciudadanía.

La práctica como acción sustancial en el aprendizaje colectivo de la ciudadanía es reconocida en comunidad a través de los siguientes aspectos. a) la práctica como significado, en la que la producción social de significado es el nivel más pertinente para hablar de práctica; b) la práctica como comunidad, presentándola como fuente de cohesión en una comunidad; c) la práctica como aprendizaje, entendida como proceso de aprendizaje en una estructura emergente y variable de forma constante; d) la práctica como límite, estableciendo acotaciones más allá de lo interno y de lo externo, de manera que se conciben dichos límites como un panorama complejo de periferias, y e) la práctica como situación, cuando ésta es parte de una comunidad, o bien comienza a ser parte de una constelación de comunidades que apuntan a diversos fines, ${ }^{16}$ como los fines a los que se adhieren los ideales de una ciudadanía mundial.

${ }^{15}$ Wenger, Comunidades de práctica, p. 71.

${ }^{16}$ Wenger, Comunidades de práctica, p. 73. 


\section{Metodología y análisis del concepto de ciudadanía mundial en jóvenes mexicanos}

Con la revisión de los conceptos clave que forman parte del acto comunicativo, y que derivan en condiciones de educación para la ciudadanía, se presentan en este apartado metodológico tres etapas de análisis que permiten establecer un panorama diagnóstico sobre el concepto de ciudadanía mundial en jóvenes mexicanos matriculados en la universidad: 1) etapa de revisión de los contenidos curriculares relacionados con ciudadanía mundial atendidos en la educación formal; 2) establecimiento de categorías que generan evidencia sobre la acepción del concepto en los sujetos observados, y 3) etapa de contraste sobre la práctica de la ciudadanía mundial y su promoción por parte de los propios jóvenes observados.

Dicha estrategia metodológica tiene base en la propuesta de Cars y West, ${ }^{17}$ quienes desarrollan un diagnóstico para evaluar los logros de la ciudadanía que apuntan al cumplimiento de los objetivos del desarrollo sostenible después de la primera década de aplicación a nivel de políticas, currículos escolares y participación civil. A pesar de que Cars y West hacen un diagnóstico situacional de los ciudadanos de Suecia (país altamente calificado en los indicadores para el desarrollo), se considera el caso debido al énfasis holístico que como resultado proponen para poder analizar el fenómeno, y es que a decir de los autores, no se puede contemplar un avance en las políticas de ciudadanía mundial con una sola arista de observación, se tiene que propiciar una mirada integradora que dé cuenta del papel de cada uno de los actores clave en la conformación social.

Se reconoce este trabajo metodológico como un trabajo de tipo exploratorio y de enfoque cualitativo, que apela a la inducción como método general para establecer las particularidades de los sujetos de estudio, y que incorpora diferentes técnicas de recolección de la

${ }^{17}$ Cars y West, "Education for Sustainable Society". 
información en el transcurso de las tres etapas del proceso. Todo lo anterior para propiciar los contrastes y relaciones que permiten dar respuestas parciales a la indagatoria central de la propia investigación.

\section{Contenidos relacionados con ciudadanía mundial en la currícula de la educación básica en México}

La educación básica establece como obligatoria una línea de saberes competentes al reconocimiento de derechos y obligaciones del ciudadano mexicano desde la incorporación de éste en la educación primaria, esto en el formato de asignaturas de la llamada formación cívica y ética, que en el caso del infante de primaria se limita al reconocimiento de deberes y prerrogativas del orden social e institucional. Es hasta la llamada educación secundaria (en los grados de segundo y tercero) en donde se comienzan a desarrollar diálogos complejos que implican la participación del educando en sociedad, y que le hacen sabedor de las condiciones de su libertad para la toma de decisiones en un marco de alteridad a favor de un bien común.

De manera explícita la Secretaría de Educación Pública declara que los propósitos de la formación cívica y ética en educación secundaria son que los alumnos:

Se reconozcan como sujetos con dignidad y derechos, capaces de tomar decisiones, y de asumir compromisos que aseguren el disfrute y cuidado de su persona, tanto en su calidad de vida personal como en el bienestar colectivo, encaminados hacia la construcción de su proyecto de vida.

Comprendan que los derechos humanos y la democracia son el marco de referencia para tomar decisiones autónomas que enriquezcan la convivencia, el cuestionar acciones que violen el derecho de las personas y afecten su ambiente natural y social. 
Reconozcan que las características de la democracia en un Estado de derecho les permiten regular sus relaciones con la autoridad, las personas y los grupos, al participar social y políticamente de manera activa en acciones que garanticen formas de vida más justas, democráticas, interculturales y solidarias. ${ }^{18}$

Como se puede apreciar, en los propósitos de la formación cívica y ética de la educación básica secundaria, no queda explícita una intencionalidad que apunta de manera directa hacia el concepto de ciudadanía mundial, o bien hacia los objetivos del desarrollo sostenible. Si bien en la declaratoria de sus propósitos el plan educativo hace énfasis en la importancia de los derechos humanos, pretende situarlos en el reconocimiento del marco del derecho y bajo una figura jurídica, lo que en el debate de la mundialización indica un sentido contrario a lo deseable en las nuevas generaciones de ciudadanos.

En este contexto de observaciones se dispuso de una criba de análisis para estudiar dos documentos curriculares clave, el Plan de estudios 2011 de la Educación Básica, y el Programa de estudio de Formación Cívica y Ética de la educación básica secundaria. La intención en el estudio de estos documentos es la de identificar aquellos conceptos trabajados para su desarrollo en el aula, y que se vinculan con las propuestas de ciudadanía mundial y de los objetivos del desarrollo sostenible. Para tales propósitos se pone el acento en algunos conceptos que aparecen en los citados documentos, en los conceptos que no aparecen y en algunos marcadores discursivos que dan idea del sentido que propone el plan de estudios para el concepto.

${ }^{18}$ SEP, Plan de estudios 2011, p. 14. 
Cuadro 1. Revisión de los planes de estudio y su orientación a una ciudadanía mundial

\begin{tabular}{|c|c|c|c|}
\hline \multicolumn{4}{|c|}{ Plan de Estudios 2011 (General) } \\
\hline GONGEPTO & $\begin{array}{c}\text { Cantidad de } \\
\text { apariciones en } \\
\text { el doc. }\end{array}$ & $\begin{array}{c}\text { Ámbito de } \\
\text { competencia } \\
\text { en que se } \\
\text { manifiesta }\end{array}$ & $\begin{array}{l}\text { Énfasis con el } \\
\text { que se emplea el } \\
\text { concepto }\end{array}$ \\
\hline Internacional & 8 & $\begin{array}{l}\text { Principalmente de } \\
\text { carácter organiza- } \\
\text { tivo orientado al } \\
\text { profesorado. }\end{array}$ & $\begin{array}{l}\text { A manera de } \\
\text { coordinación } \\
\text { con estándares } \\
\text { internacionales } \\
\text { regulatorios. }\end{array}$ \\
\hline Mundial & 10 & $\begin{array}{l}\text { Ámbito cognitivo, } \\
\text { aislado de posibili- } \\
\text { dades de empatía o } \\
\text { reflexión }\end{array}$ & $\begin{array}{c}\text { Aparece en formato } \\
\text { de sustantivo } \\
\text { (mundo), como } \\
\text { referencia de } \\
\text { regionalidad. }\end{array}$ \\
\hline Global & 1 & Ámbito cognitivo & $\begin{array}{l}\text { Aparece como adje- } \\
\text { tivo acompañando } \\
\text { el sustantivo; } \\
\text { "mundo global". }\end{array}$ \\
\hline Ciudadanía & 2 & Ámbito cognitivo & $\begin{array}{l}\text { En una ocasión } \\
\text { aparece como } \\
\text { sustantivo (ciuda- } \\
\text { dano), y en otra } \\
\text { como adjetivo } \\
\text { (valores ciudadanos). }\end{array}$ \\
\hline Equidad & 4 & $\begin{array}{c}\text { Cognitivo y } \\
\text { socioemocional }\end{array}$ & $\begin{array}{l}\text { La equidad sí se } \\
\text { manifiesta como } \\
\text { un estadio de ideal } \\
\text { en búsqueda. }\end{array}$ \\
\hline Pluralidad & 12 & $\begin{array}{c}\text { Cognitivo y } \\
\text { socioemocional }\end{array}$ & $\begin{array}{l}\text { Tiene énfasis en } \\
\text { la promoción } \\
\text { y riqueza de la } \\
\text { pluralidad, sobre } \\
\text { todo en el sentido } \\
\text { lingüístico. }\end{array}$ \\
\hline Intercultural & 2 & $\begin{array}{c}\text { Cognitivo y } \\
\text { socioemocional }\end{array}$ & $\begin{array}{l}\text { Se manifiesta } \\
\text { como un fenómeno } \\
\text { complejo y nece- } \\
\text { sario en los ideales } \\
\text { educativos. }\end{array}$ \\
\hline Derechos Humanos & - & - & $\begin{array}{l}\text { Solamente hace } \\
\text { referencia a derechos } \\
\text { de los connacionales. } \\
\text { No se propician } \\
\text { directrices al } \\
\text { respecto. }\end{array}$ \\
\hline
\end{tabular}




\begin{tabular}{|c|c|c|c|}
\hline \multicolumn{4}{|c|}{ Programa de Estudio de Formación Cívica y Ética } \\
\hline CONGEPTO & $\begin{array}{c}\text { Cantidad de } \\
\text { apariciones en } \\
\text { el doc. }\end{array}$ & $\begin{array}{l}\text { Ámbito de } \\
\text { competencia } \\
\text { en que se } \\
\text { manifiesta }\end{array}$ & $\begin{array}{l}\text { Énfasis con el } \\
\text { que se emplea el } \\
\text { concepto }\end{array}$ \\
\hline Internacional & 1 & Ámbito cognitivo & $\begin{array}{l}\text { Se declara como } \\
\text { propósito de } \\
\text { comprensión para } \\
\text { el educando }\end{array}$ \\
\hline Mundial & - & - & $\begin{array}{l}\text { Aparece solamente } \\
\text { el sustantivo mundo. }\end{array}$ \\
\hline Global & 3 & Ámbito cognitivo & $\begin{array}{l}\text { En dos ocasiones } \\
\text { aparece como adje- } \\
\text { tivo, pero en una sí } \\
\text { sugiere la impor- } \\
\text { tancia de procesos } \\
\text { de globalización. }\end{array}$ \\
\hline Ciudadanía & 10 & $\begin{array}{l}\text { Cognitivo, socioe- } \\
\text { mocional y } \\
\text { actitudinal }\end{array}$ & $\begin{array}{l}\text { La ciudadanía sí es } \\
\text { presentada como } \\
\text { ideal a alcanzar, y } \\
\text { hace mucho énfasis } \\
\text { del marco jurídico } \\
\text { para la participa- } \\
\text { ción ciudadana. }\end{array}$ \\
\hline Equidad & 13 & $\begin{array}{l}\text { Cognitivo, socioe- } \\
\text { mocional y } \\
\text { actitudinal }\end{array}$ & $\begin{array}{l}\text { La equidad sí se } \\
\text { manifiesta como } \\
\text { un estadio de } \\
\text { ideal en búsqueda. } \\
\text { Menciona marca- } \\
\text { dores relacionados } \\
\text { con la empatía. }\end{array}$ \\
\hline Pluralidad & 2 & Ámbito cognitivo & $\begin{array}{l}\text { En los dos casos } \\
\text { asimilable al ejer- } \\
\text { cicio de democracia. } \\
\text { Aparece también el } \\
\text { adjetivo plural. }\end{array}$ \\
\hline Intercultural & 2 & Ámbito cognitivo & $\begin{array}{c}\text { En ambos casos } \\
\text { como adjetivo. }\end{array}$ \\
\hline Derechos Humanos & 24 & $\begin{array}{l}\text { Cognitivo, socio- } \\
\text { emocional y } \\
\text { actitudinal }\end{array}$ & $\begin{array}{c}\text { Se propone } \\
\text { ampliamente el } \\
\text { concepto desde } \\
\text { diferentes aristas } \\
\text { para su desarrollo } \\
\text { como contenido } \\
\text { temático. }\end{array}$ \\
\hline
\end{tabular}




\section{Categorías determinantes para la relación de la ciudadanía mundial con las currícula de la educación básica}

Debido a que, en el estudio de los documentos de la Secretaría de Educación Pública para la educación básica en México, no se observa un tratamiento directo de las temáticas orientadas a la ciudadanía mundial, y como parte de la estrategia metodológica, se establece en este apartado un marco de categorías analíticas que pueden dar evidencia de que el educando incorpora a su vida saberes relacionados con el concepto en cuestión. En este sentido se parte de la noción de la propia unEsco para la ciudadanía mundial, cuando la describe como:

Un paradigma marco en el que se narran de forma resumida las formas en que la educación puede desarrollar los conocimientos, las competencias, los valores y las actitudes que los estudiantes necesitan para garantizar un mundo más justo, pacífico, tolerante, inclusivo y sostenible. ${ }^{19}$

Así, con esta línea discursiva se retoman los planes de estudio de la formación cívica y ética en México, y se establecen las siguientes categorías de análisis, las cuales de acuerdo con lo expuesto en la currícula escolar podrían permitir la apreciación de los saberes relacionados con la ciudadanía mundial y que son apropiados por los egresados de dicha currícula, es decir, en los jóvenes que son objeto del presente estudio. Dichas categorías, detectadas y estructuradas, que fungen como eje nodal en el contraste desarrollado en la tercera etapa metodológica, se presentan a continuación.

A) Categorías del ámbito cognitivo que el propio programa de estudios propicia a través del trabajo de sus contenidos temáticos:

\footnotetext{
${ }^{19}$ UNESCO, Educación para la ciudadanía mundial, p. 9.
} 
Sistemas, estructuras y procesos mundiales, principalmente referente a procesos de gobernanza local, nacional y mundial, derecho humanitario, ciudadanía para la paz, derechos humanos, Estado de derecho y la noción de empresas trasnacionales.

Condiciones de alcance mundial identificados en contenidos vinculados con globalización, inmigración, relaciones fronterizas, interconexiones, interdependencia, pobreza mundial, desigualdad mundial, genocidio, intolerancia, terrorismo, guerra, enfermedades epidémicas del siglo xxi, refugiados, cambio climático, patrimonio humanitario, desarrollo sostenible.

B) Categorías del ámbito socioemocional identificadas en el programa de estudios a través de la cobertura de sus contenidos:

Identidades múltiples en referencia y desarrollo de la noción del individuo y los demás, la comunidad, el país, el mundo, identidad personal, colectiva, cultural, nacional, regional y mundial, el país y la vida social en constante negociación en búsqueda del bien común.

Diferencias y respeto por la diversidad, en donde se contemplan temas como la empatía, el diálogo, el respeto, la solidaridad y la tolerancia interculturales, la discriminación y el racismo. Todos los anteriores considerados como posibles valores para la ciudadanía mundial.

G) Categorías del ámbito conductual identificadas principalmente en las estrategias didácticas y actividades complementarias para la atención de los contenidos del programa:

Compromiso, participación, acciones, tales como la participación actual y futura en protestas cívicas por cuestiones mundiales, información y debates sobre cuestiones sociopolíticas mundiales y la acción directa sobre cuestiones de alcance mundial, principalmente aquellas que propician la incorporación de las Tic. 


\section{Práctica y promoción de la ciudadanía mundial en jóvenes mexicanos}

En el presente apartado se presenta la estrategia mediante la cual se recolectó la información referida por parte de los propios jóvenes en relación con su dominio y práctica de los valores asimilables a la ciudadanía mundial. La estrategia se desarrolló mediante grupos de discusión convocados en seis recintos universitarios de índole pública y privada. Se seleccionaron a los jóvenes de nuevo ingreso en los programas de licenciatura de la promoción de verano de 2017; lo anterior, debido a que son estos jóvenes quienes recibieron su instrucción de educación básica secundaria ya con apego al Plan de estudios 2011 de la Secretaría de Educación Pública en México.

Los jóvenes universitarios participantes sesionaron grupos de discusión en las siguientes universidades: Universidad Autónoma Metropolitana (Ciudad de México), Universidad Pedagógica Nacional (Ciudad de México), Universidad Michoacana de San Nicolás de Hidalgo (Morelia, Michoacán), Universidad La Salle (León, Guanajuato), Tecnológico de Estudios Superiores de Monterrey (Monterrey, Nuevo León) y Universidad Vasco de Quiroga (Morelia, Michoacán), el promedio de participación fue de 11.5 alumnos por cada grupo de discusión, con un total de 69 concurrentes. Todos los participantes sesionaron de forma voluntaria y en una locación de su universidad de procedencia. Los grupos de discusión se desarrollaron en el periodo comprendido entre septiembre de 2017 y febrero de 2018.

Las dinámicas de las sesiones de discusión se coordinaron para desarrollar desde la perspectiva de los participantes y bajo la coordinación de un monitor alumno integrante del grupo. Se les pidió a los alumnos que plantearan discusiones que incluyeran sus opiniones por espacio de una hora, y máximo dos horas, en donde se abordaron los temas clave tocando de manera medular las categorías analíticas identificadas en los documentos estudiados de la Secretaría de Educación Pública presentados en el subapartado 3.2 del mismo artículo. De esta manera se les dotó a los monitores de discusión con un guión 
estructurado para más tarde, mediante dispositivos de registro, para posibilitar el análisis discursivo orientado a las primeras inferencias, en concordancia con el objetivo de la investigación.

El guión de las discusiones, se atendió en orden cronológico por la mayoría de los participantes y se propició alrededor de las siguientes temáticas:

A. Acepción personal y grupal del concepto "Ciudadanía Mundial".

B. Sistemas, estructuras y procesos mundiales.

C. Condiciones de alcance mundial identificados en contenidos vinculados con globalización, inmigración, relaciones fronterizas.

D. Identidades múltiples en referencia y desarrollo de la noción del individuo y los demás.

E. Diferencias y respeto por la diversidad.

F. Compromiso, participación, acciones, tales como la participación actual y futura en protestas cívicas por cuestiones mundiales.

G. Referencias de cursos escolarizados para el aprendizaje y promoción de los conceptos discutidos.

H. Referencias distintas al espacio escolarizado en donde han encontrado cabida a sus preocupaciones relacionadas con los valores de la "Ciudadanía Mundial".

Una vez registradas las seis sesiones con el total de los participantes, se procedió con un análisis discursivo de las discusiones, entre las cuales se generaron las relaciones destacables que permiten sugerir la apropiación de los conceptos relacionados con la ciudadanía mundial, así como las modalidades mediante las cuales los jóvenes mexicanos las dicen practicar y promover.

\section{Conclusiones}

Como resultado del contraste entre las currícula escolares propuestas por la Secretaría de Educación Pública en México y las referencias que los propios jóvenes observados otorgan, en relación con los 
conceptos vinculados a la ciudadanía mundial, se presentan algunas inferencias preliminares y nuevas indagatorias que se desprenden como resultado del trabajo de investigación. En esta primera entrega los resultados se obtienen solamente del tratamiento con algunos jóvenes, considerados particularmente; no obstante, los resultados permitirán establecer líneas estratégicas para revisar el caso de otros jóvenes en condiciones diversas.

Se tiene que en los documentos de "Plan de estudios 2011" y "Programa de estudios para la Formación Cívica y Ética", la Secretaría de Educación Pública en México sí hace referencia a los conceptos relacionados con la ciudadanía mundial. No trata el tema directamente, como tampoco lo hace sobre los objetivos del desarrollo sostenible; sin embargo, al indagar sobre sus propuestas cognitivas, socioemocionales y actitudinales que promueve en el estudiantado, sí se aprecia una relación directa con estos ideales establecidos como rumbo para los indicadores de desarrollo humano. Situados a más de diez años de suscritos los acuerdos entre los países de América Latina y las instituciones internacionales, para la incorporación de contenidos relacionados con la ciudadanía mundial y los objetivos para el desarrollo sostenible en las currícula escolares, es pertinente la nueva revisión y la evaluación para que autoridades, instituciones, gobiernos e instancias competentes identifiquen los resultados hasta ahora obtenidos y, en su caso, las mejoras a incorporar.

En los grupos de discusión se pudo apreciar de manera generalizada que los jóvenes sí identifican las características de lo que se concibe como ciudadanía mundial, sin embargo, no lo saben definir ni ubican con precisión algún documento de apoyo en el cual puedan tener certeza sobre el concepto. También identifican algunas instancias internacionales que promueven la noción de ciudadanía mundial, principalmente la UNESCO, y todos refieren no recordar alguna exposición y/o discusión en este sentido durante su estancia en la educación básica. Aunado a lo anterior sí reconocen que a través del curso de formación cívica y ética comprendieron algunas lógicas de estructura 
y gobierno, principalmente locales, además de reconocer la función de algunas instituciones regulatorias en el mundo.

La actividad registrada en las sesiones de discusión también da evidencia de la preocupación y atención que los jóvenes tienen sobre los temas de alcance mundial, principalmente los relacionados con procesos de paz, de migración y de relaciones fronterizas. Destacan las crisis de gobernabilidad para la seguridad en regiones específicas como Medio Oriente y África, dato que llama la atención, ya que al ser jóvenes mexicanos parecen no considerar en este rubro las condiciones de Latinoamérica. Sí manifiestan una noción del individuo en función del otro, sin embargo, dicha noción es mediada por el concepto de nacionalidad y extranjerismo, en el discurso se aprecia una condición de calificar como distantes los problemas de otros en su lucha por la paz y la seguridad. La mayoría de estas referencias son asimiladas por los jóvenes a través de los medios informativos, así como lo reconocen ellos mismos.

La diversidad y la participación ciudadana son conceptos más cercanos para los jóvenes universitarios en observación, la mayoría de los ejemplos socializados se sitúan en el plano local; aquí se aprecia mucha diferencia entre los jóvenes participantes en la capital del país y aquellos de las ciudades de Morelia, Monterrey y León. La diversidad en las preferencias sexuales y condiciones de subactivismo político fueron las líneas discursivas más desarrolladas, con muchas más referencias contextuales los jóvenes de la capital; sin embargo, con poco sustento en documentos normativos y con mucho acervo de experiencias cercanas. En este rubro temático, nuevamente los jóvenes dejan fuera la discusión del espacio escolarizado; apuntan nunca haber discutido el tema como parte de las actividades en el aula, por el contrario, otorgan mucho valor a su participación de socialización entre pares en entornos asumidos como propios y principalmente mediados a través de las tecnologías de la información y comunicación. Señalan que es en los entornos, principalmente los virtuales, en los que abiertamente se debaten los significados de estos conceptos, y es también en donde han encontrado y promovido mucha solidaridad al respecto. 
Se aprecia que en las nuevas generaciones de ciudadanos mexicanos, los matriculados en la educación superior, sí hay una conciencia de valores asimilables al concepto de "Ciudadanía Mundial", ellos mismos reconocen construir estas referencias en su competencia cognitiva, y en algunos casos hasta socioemotiva, gracias a las experiencias atendidas en el aula a lo largo de su trayectoria en los sistemas de educación básica, principalmente las condiciones de ejercicio y convivencia estructural determinada por los gobiernos, las instituciones y las condiciones de responsabilidad civil. En continuidad con esta perspectiva, los mismos jóvenes otorgan evidencia de adquirir competencias actitudinales hacia los valores de la ciudadanía mundial en espacios fuera del entorno escolarizado, y en algunos casos dicen fomentar la práctica de dichos valores a través de las convocatorias, y las solidaridades, que encuentran en los escenarios asumidos como propios, principalmente en temas de diversidad, de garantías individuales y de subactivismo político. Finalmente, los jóvenes mexicanos asimilan el concepto de "Ciudadanía Mundial" como una categoría complicada para su definición y aprendizaje, pero al mismo tiempo le otorgan valoraciones positivas identificando a ésta como un ideal a seguir que deberá permanecer en constante significación y reconstrucción. 


\section{Siglas}

Gepal Comisión Económica para América Latina y el Caribe

ods Objetivos para el Desarrollo Sostenible

ocDE Organización para la Cooperación y el Desarrollo Económico

onu Organización de las Naciones Unidas

PNUD Programa de las Naciones Unidas para el Desarrollo

SEP Secretaría de Educación Pública

unEsco Organización de las Naciones Unidas para la Educación la Ciencia y la Cultura

\section{Fuentes}

\section{Bibliografía}

Cars M. y West E., "Education for Sustainable Society: Attainments and Good Practices in Sweden During the United Nations Decade for Education for Sustainable Development (UNDESD)", en Environment, Development and Sustainability, vol. 17, núm. 1, 2015, pp. 1-22.

Freire, P., Pedagogía del oprimido (J. Mellado, Trad.), México, Siglo xxi Editores, 2005.

Fernández, C. y Galguera, L., Teorías de la comumicación, México, McGraw-Hill, 2009.

PNud, Panorama general. Informe sobre el desarrollo humano 2016. Desarrollo humano para todos, Nueva York, PNUD, 2016.

Reimers, DeShano y Trevino, Where is the "Education" in Conditional Cash Transfers in Education?, Montreal, unesco Institute for Statistics, 2006.

Rizo, M. "Interacción y comunicación en entornos educativos: Reflexiones teóricas, conceptuales y metodológicas", en revista E-Compós, abril 1-16 [consultado en http://www.compos.org.br/ seer/index.php/e- compos/article/viewFile/143/144]. 
Rodríguez, J. L., "Comunidades virtuales, práctica y aprendizaje: elementos para una problemática", en Revista Electrónica Teoría de la Educación. Educación y Cultura en la Sociedad de la Información, Vol. 8, núm. 3, 2007, pp. 6-22.

Sarramona, J., Vázquez, G., y Colom, C. A. J., Educación no formal, Barcelona, Ariel, 1998.

Sirvent, M. T., "La educación de jóvenes y adultos frente al desafío de los movimientos sociales emergentes en Argentina", en Revista Brasileira de Educaçao, núm. 28, 2005, pp. 37-49.

SEP, Plan de estudios 2011, México, Secretaría de Educación Pública, 2011.

SEP, Programas de estudio 2011. Educación básica secundaria. Formación cívica y ética, Mexico, Secretaría de Educación Pública, 2011.

Tawil, S., La educación para la "ciudadanía mundial": marco para el debate. Investigación y Prospectiva en Educación, París, unesco, 2013.

unesco, Conceptos de ciudadanía mundial integrados a los lineamientos curriculares de 10 paises: análisis comparativo, Ginebra, Suiza, unEsco-Oficina Internacional de Educación, 2017.

unesco, Educación para la ciudadanía mundial. Temas y objetivos de aprendizaje, París, unesco, 2015.

Wenger, E., Comunidades de práctica: Aprendizaje significado e identidad (G. Sánchez, trad.), Barcelona, Paidós Ibérica, 2001.

\section{Electrónicas}

ocde, Panorama de la educación 2015. ocde [documento electrónico] disponible en: https://www.oecd.org/mexico/Education-at-aglance-2015-Mexico-in-Spanish.pdf [consultado el 22 de marzo de 2018]. 